\title{
CIRCADIAN RHYTHMICITY IN THE OCCURRENCE OF SPONTANEOUS ACUTE DISSECTION AND RUPTURE OF THORACIC AORTA
}

\author{
Massimo Gallerani, MD, ${ }^{\mathrm{a}}$ Francesco Portaluppi, MD, ${ }^{\mathrm{b}}$ Enrico Grandi, MD, ${ }^{\mathrm{c}}$ and Roberto Manfredini, ${ }^{\mathrm{b}}$ Ferrara, Italy
}

Much evidence is available on the existence of a circadian variability in the occurrence of many cardiovascular events. Myocardial ischemia and infarction, sudden cardiac death, acute pulmonary thromboembolism, and cerebrovascular diseases all show the highest rate of occurrence during the morning hours. ${ }^{1,2}$ Among the acute unfavorable events of the cardiovascullar system, the spontaneous dissection or rupture of thoracic aorta often plays a dramatic and most frequently fatal role. This study investigated whether a specific circadian pattern exists for these spontaneous acute events of the thoracic aorta.

Subjects and methods. All patients referred to the St. Anna Hospital of Ferrara, Italy, from January 1985 to December 1994 for a spontaneous acute event of the thoracic aorta were considered. Diagnosis was ascertained by clinical examination (severe chest pain, acute neurologic signs, signs of massive hemorrhage, or a combination of these) and instrumental tests as possible in light of the conditions of emergency (computed tomography and angiography); it was then confirmed at surgical intervention and by necropsy in all cases of death.

Data analysis was performed on the whole study population and in subgroups according to sex, age (younger or older than 70 years), and type of event by DeBakey's classification. Each event was categorized according to the clock hour and date of symptom onset into 24 1-hour increments (e.g., 6:00 AM to 6.59 AM was reported as 6 hours). After

From the Emergency Department, St. Anna Hospital, ${ }^{a}$ and the First Institute of Internal Medicine ${ }^{b}$ and Institute of Pathologic Anatomy, ${ }^{\mathrm{c}}$ University of Ferrara, Ferrara, Italy.

Received for publication April 1, 1996; accepted for publication July 25, 1996.

Address for reprints: Massimo Gallerani, MD, Emergency Department, St. Anna Hospital, Corso Giovecca 203, I-44100 Ferrara, Italy.

J Thorac Cardiovasc Surg 1997;113:603-4

Copyright (C) 1997 by Mosby-Year Book, Inc.

$0022-5223 / 97 \$ 5.00+0 \quad \mathbf{1 2 / 5 4 / 7 6 9 2 2}$ checking for significant variations in time with one-way analysis of variance, partial Fourier series with up to six harmonics (periods of $24,12,8$, $6,4.8$, and 4 hours) were applied to the time series. The best-fitting curves were used to assess rhythmicity. ${ }^{3}$

The combination of harmonics that best explained the variance of data was determined. We calculated the percentage of rhythm (percentage of overall data variability, a measure of goodness of the fit), the $p$ value of the $F$ statistic (indicating the statistical significance of the rhythmic function), and the amplitude (half the distance between the absolute maximum and minimum of the function) of each harmonic and the overall best-fitting curve. The midline estimating statistic of rhythm (the rhythm-adjusted 24-hour mean) and the peak and trough times of the fitted curve (times of occurrence of the absolute maximum and minimum) were also calculated, together with the acrophase of each single harmonic (peak time of rhythmic change). Significance was always assumed for $p$ values lower than 0.05 .

Results. In the 10-year period analyzed, 70 cases of spontaneous acute events of the thoracic aorta were observed. Of the affected patients, $54(77.1 \%)$ died suddenly in the emergency department and 16 $(22.9 \%)$ underwent urgent surgical intervention.

Table I. Characteristics of the study population

\begin{tabular}{lrcc}
\hline & \multicolumn{2}{c}{ Cases } & Man age $+S E$ \\
\cline { 2 - 3 } & $n$ & $\%$ & $(y r)$ \\
\hline Total population & 70 & 100.0 & $68.5 \pm 14.2$ \\
$\quad$ Male & 40 & 57.1 & $67.2 \pm 15.4$ \\
Female & 30 & 42.9 & $70.2 \pm 12.5$ \\
Aortic dissection & 63 & 90 & $67.6 \pm 14.4$ \\
Aneurysm rupture & 7 & 10 & $76.1 \pm 10.9$ \\
Type I & 36 & 51.4 & $70 \pm 12.7$ \\
Type II & 26 & 37.2 & $69 \pm 12.8$ \\
Type III & 8 & 11.4 & $60 \pm 22.4$ \\
Marfan syndrome & 2 & 2.8 & $38.5 \pm 23.3$ \\
Cystic medionecrosis & 3 & 4.3 & $42.6 \pm 27.8$ \\
\hline
\end{tabular}

$S E$, Standard error. 


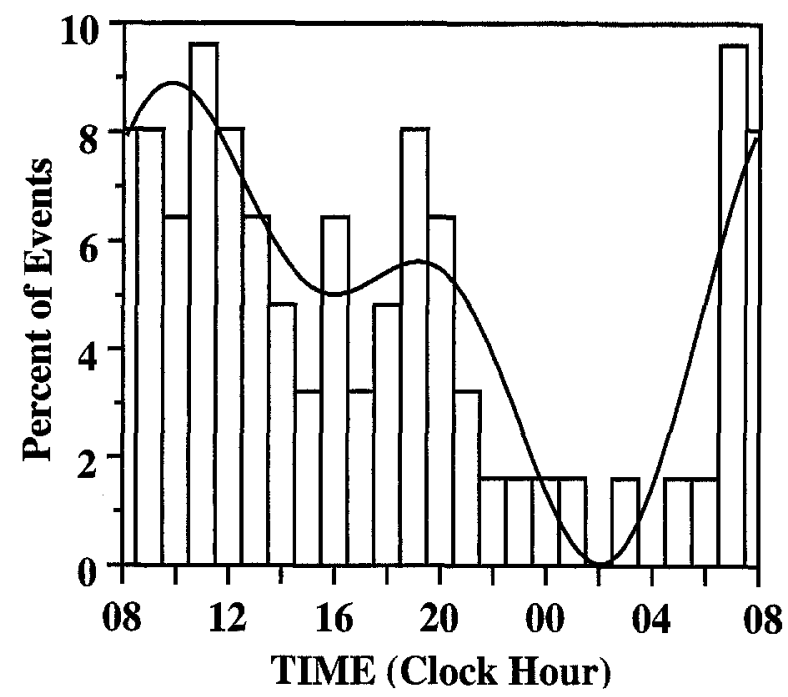

Fig. 1. Circadian distribution in study population of spontaneous acute events (dissection and rupture) of thoracic aorta. Superimposed is the best-fitting curve at Fourier analysis.

The demographic, clinical, and pathologic features of the sample population are reported in Table I.

Time of symptom onset was accurately defined in 67 cases $(95.7 \%)$. The three cases $(4.3 \%)$ with undefined time of onset were excluded from the analysis.

The circadian distribution of spontaneous acute events of the thoracic aorta among the total study population is shown in Fig. 1. At analysis of variance, the temporal distribution of the events through the 24 hours of the day showed a significant $(p<0.001)$ pattern. A primary peak was evident at around 10 hours, a secondary peak was evident at around 20 hours, and a nadir was evident during the nocturnal hours. At analysis of rhythmicity, the best-fitting curve had two significant components with periods of 24 and 12 hours, respectively. Closely similar patterns were demonstrated in the subgroups defined by age, sex, and classification type.

Discussion. The main finding of this study is the demonstration that spontaneous, thoracic aortic rupture occurs with a circadian pattern. We found a primary peak of onset in the morning hours (at around 10 hours) and a secondary peak in the evening (at around 20 hours). To our knowledge, no previous study on this subject is available in the literature. Blood pressure is likely to play a major role in the determination of these findings; the circadian pattern of blood pressure is strikingly similar to that found in all acute cardiovascular events. ${ }^{1,2}$ An association between hourly episodes of subarachnoid hemorrhage and hourly blood pressure values of ambulant normotensive and hypertensive subjects has been reported, ${ }^{4}$ suggesting that blood pressure peaks during the waking hours may also be crucial in determining the time of acute dissection and rupture of a critically weakened aortic wall. In addition to the absolute values, distinctive importance has been attributed to the impact on the arterial wall of rapid changes in the blood pressure. ${ }^{5}$ This is consistent with the observation that blood pressure variability may be a determinant of vascular organ damage independently of the mean 24 -hour blood pressure. ${ }^{6}$ Blood pressure variability is bound to be higher during the day than during the night as a consequence of the varying external stimuli and physical and mental activities, which explains the lower frequency that we found of spontaneous, acute aortic events during sleep.

To precisely assess the time frame of our cases, we excluded spontaneous aortic events that did not have acute signs clearly evident at the time of onset. Our findings therefore cannot be extended to chronic ruptures and dissections. Because of the clinical selection we had to make, some acute cases may have been excluded from our series (in the few instances in which the clinical signs of an external leak of the aorta could have remained unnoticed for some time), but their negligible number would not likely have influenced our results significantly.

\section{REFERENCES}

1. Gallerani M, Manfredini R, Ricci L, et al. Chronobiological aspects of acute cerebrovascular diseases. Acta Neurol Scand 1993;87:482-7.

2. Manfredini R, Gallerani M, Portaluppi F, Fersini C. Relationships of the circadian rhythms of thrombotic, ischemic, hemorrhagic, and arrhythmic events to blood pressure rhythms. Ann N Y Acad Sci 1996;83:141-58.

3. Mojón A, Fernández JR, Hermida RC. Chronolab: an interactive software package for chronobiologic time series analysis written for the Macintosh computer. Chronobiol Int 1992;9: 403-12.

4. Fogelholm RR, Turjanmaa VM, Nuutile MT, Murros KE, Sarna S. Diurnal blood pressure variations and onset of subarachnoid haemorrhage: a population-based study. J Hypertens 1995;13:495-8.

5. Hossmann V. Circadian changes of blood pressure and stroke. In: Zülch KJ, editor. Cerebral circulation and stroke. New York: Springer, 1971:203-8.

6. Frattola A, Parati G, Cuspidi C, Albini F, Mancia G. Prog nostic value of 24 -hour blood pressure variability. $J$ Hypertens 1993;11:1133-7. 